DOI: $10.5216 /$ cab.v14i4.17693

\title{
EFICIÊNCIA REPRODUTIVA E DINÂMICA FOLICULAR DE ÉGUAS CAMPOLINA DE ACORDO COM A CONDIÇÃO CORPORAL
}

\author{
CARMEM ESTEFÂNIA SERRA NETO ZÚCCARI ${ }^{1}$, ÉRIKA SALTIVA CRUZ BENDER ${ }^{2}$, Eliane Vianna Costa \\ E Silva ${ }^{1}$, Helton MatTana SATURNinO ${ }^{3}$ \\ ${ }^{1}$ Professoras Doutoras da Universidade Federal do Mato Grosso do Sul, Campo Grande, MS, Brasil. \\ carmem.zuccari@ufms.br \\ ${ }^{2}$ Pós Graduanda da Universidade Federal do Mato Grosso do Sul, Campo Grande, MS, Brasil. \\ ${ }^{3}$ Professor Doutor da Universidade Federal de Minas Gerais, Belo Horizonte, MG, Brasil.
}

O presente estudo teve como objetivo avaliar o efeito da condição corporal (CC) sobre a eficiência reprodutiva e a dinâmica folicular de éguas, no decorrer da estação de monta. Foram acompanhadas 134 éguas (92 não lactantes e 42 lactantes) que, em novembro e dezembro, foram submetidas à inseminação artificial e, de janeiro a março, à monta natural controlada, em dias alternados. Mantidas sob rufiação diária em grupo e a campo, quando o cio era detectado, iniciou-se o controle folicular diário por palpação trans-retal até a detecção da ovulação. O escore da CC foi avaliado nos meses de agosto, novembro, janeiro e março. A taxa de anestro foi $26,11 \%$ e a de gestação $56,7 \%$, mas, considerando-se apenas as éguas cíclicas, $77 \%$ tornaram-se gestantes. O número de ciclos/concepção foi 2,3 e de saltos/ciclo 2,1. Houve efeito $(\mathrm{P}<0,05)$ da evolução da $\mathrm{CC}$ sobre o número de éguas cíclicas e a taxa de gestação. $\mathrm{O}$ mês da estação de monta e a CC influenciaram todas as características foliculares e a taxa de gestação $(\mathrm{P}<0,05)$. Concluiu-se que a condição corporal tem efeito sobre a atividade cíclica, a dinâmica folicular e a fertilidade de éguas.

PALAVRAS-CHAVE: Equino; escore corporal; taxa de gestação.

\section{REPRODUCTIVE EFFICIENCY AND FOLLICULAR DYNAMIC OF CAMPOLINA MARES ACCORDING TO BODY CONDITION}

\section{ABSTRACT}

The aim of this study was to evaluate the effect of body condition (BC) on the reproductive efficiency and follicular dynamics of mares during the breeding season. We evaluated 134 mares (92 broodmares and 42 lactating) that were submitted to artificial insemination in November and December, and then put under natural controlled breeding, in alternating days, from January to March. They were kept under daily heat detection in group at field, so that at the moment it was detected, daily follicular control was initiated by rectal palpation until the detection of ovulation. The $\mathrm{BC}$ was evaluated in the months of August, November, January and March. Anestro rate was $26.11 \%$ and pregnancy rate was $56.7 \%$; however, when considering only the cyclic mares, it was $77 \%$. The number of cycles/conception was 2.3 and of mounts/cycle 2.1. The BC evolution affected $(\mathrm{P}<0.05)$ the number of cyclic mares and the pregnancy rate. The month of breeding season and the BC influenced all follicular characteristics and pregnancy rate $(\mathrm{P}<0.05)$. We could conclude that the body condition affects the mare's cyclical activity, follicular dynamics and fertility.

KEYWORDS: Body score; equine; pregnancy rate. 


\section{INTRODUÇÃO}

A eficiência reprodutiva é um dos fatores que melhor traduzem a adequação da técnica de exploração empregada em um plantel, pois é a resultante da interação de inúmeras variáveis. Para a obtenção de uma elevada eficiência reprodutiva, a literatura tem evidenciado a importância de um bom escore de condição corporal em éguas (BUFF et al., 2002; GENTRY et al., 2002ab; GASTAL et al., 2004; CAVINDER et al., 2007; RODRIGUES et al., 2011). Para avaliar a condição corporal (CC) pesquisadores desenvolveram diferentes técnicas visando a obter maior confiabilidade e precisão (HENNEKE et al., 1983; GENTRY et al., 2004; CARTER et al., 2009); entretanto, o procedimento mais aceito e utilizado na experimentação de equinos é o sistema criado por HENNEKE et al. (1983), no qual se atribui aos animais um escore que varia de 1 a 9, sendo o escore 1 referente ao animal emaciado e o escore 9 àquele obeso.

FERREIRA-DIAS et al. (2005), ao considerarem o peso corporal de animais da raça Lusitana, relataram que, em média, éguas com maior peso corporal $(491-590 \mathrm{Kg})$ retomaram a atividade ovariana antes do equinócio de primavera, enquanto aquelas com menor peso corporal (390-490 Kg) voltaram a ovular mais tardiamente, já na primavera. No mesmo sentido, VECCHI et al. (2010) observaram que éguas não lactantes da raça Standardbred com boa CC tiveram a primeira ovulação mais precocemente que éguas com má condição corporal e RODRIGUES et al. (2011) constataram que éguas doadoras de embriões com $\mathrm{CC}<6,5$ apresentaram menor diâmetro do folículo dominante. PEUGNET et al. (2010) verificaram que éguas com boa CC apresentaram intervalo médio entre ovulações menor (23 dias) que aquelas em má CC (28,4 dias).

O presente estudo teve como objetivo avaliar o efeito da condição corporal de éguas da raça Campolina, mantidas em regime extensivo de criação, sobre a ocorrência de atividade cíclica, a dinâmica folicular e a eficiência reprodutiva durante a estação de monta.

\section{MATERIAL E MÉTODOS}

O estudo foi conduzido no ano de $1990 \mathrm{em}$ propriedade rural localizada a uma altitude de $478,37 \mathrm{~m}$, tendo como coordenadas geográficas os paralelos de $17^{\circ} 35^{\prime} 08^{\prime \prime}$ de latitude sul e 444' $43^{\prime}$ " de longitude oeste de Greenwich. De acordo com KÖEPPEN (1931), o clima da região pode ser classificado como do tipo Aw, caracterizado por inverno seco e verão chuvoso. A temperatura média da região durante o período experimental (agosto a março) foi de $25^{\circ} \mathrm{C}$ e a precipitação pluviométrica de $643,6 \mathrm{~mm}$.

De agosto a dezembro, as éguas foram mantidas em pastos de Brachiaria humidicola Jack, nos quais foram postos regularmente rolos de feno da mesma gramínea. De janeiro a março, as éguas permaneceram em pastos de Andropogon gayanus Hack var. bisquamulatus. Forneceu-se água e sal mineral ad libitum.

A CC foi estimada de acordo com o sistema de escore estabelecido por HENNEKE et al. (1983), cuja escala varia de 1 a 9. Para a análise estatística dos dados, foram definidas três classes de CC: Classe 1 - escore $\leq 3,5$; Classe 2 - escore de 4 a 4,5; Classe 3 - escore $\geq 5$.

Do manejo reprodutivo participaram 134 éguas da raça Campolina com idade média de 7,5 $\pm 3,2$ anos, mantidas em regime extensivo de criação, enquanto os garanhões, adultos e de fertilidade conhecida, permaneceram estabulados. Do total de éguas, $42(31,34 \%)$ estavam gestantes e as parições ocorreram de agosto a março e 92 $(68,65 \%)$ estavam não gestantes. A estação de monta (EM) transcorreu de 15 de novembro a 31 de março, sendo $81 \%$ das éguas cobertas por dois reprodutores e as demais por um terceiro garanhão.

A detecção do estro se fez através da rufiação diária, em grupo e a campo, sendo o rufião e as éguas mantidos em piquetes adjacentes e sob observação. As éguas identificadas em estro foram submetidas ao controle folicular diário, por palpação trans-retal, até a detecção da ovulação. A inseminação artificial ou monta natural controlada tinha início quando se detectava a presença de um folículo com $\geq 35 \mathrm{~mm}$ de diâmetro.

Durante os primeiros 45 dias da EM as éguas foram inseminadas, em dias alternados, com sêmen a fresco diluído com Meio de Mínima Contaminação - M.M.C. (KENNEY et al., 1975), mediante a deposição de 500 x $10^{6}$ de espermatozoides no corpo do útero. Após esse 
período, as éguas foram submetidas ao regime de monta natural controlada, em dias alternados.

Fez-se a avaliação do crescimento folicular, mediante palpação trans-retal diária durante o estro, estimando-se os seguintes parâmetros: número de folículos pequenos (NFPEQ - $\leq 15 \mathrm{~mm}$ ); número de folículos médios (NFMED - > 15 a $<35 \mathrm{~mm}$ ); número de folículos grandes (NFGRA - $\geq 35 \mathrm{~mm}$ ); número total de folículos (NFOLS = NFPEQ + NFMED + NFGRA) e diâmetro do maior folículo (MAIORF)

O diagnóstico de gestação se fez mediante palpação trans-retal aos 20 dias após a ovulação e foi confirmado aos 30, 45 e 60 dias.

Nas éguas que não apresentaram estro até janeiro, as quais eram submetidas à palpação trans-retal a intervalos de três dias, administrou-se prostaglandina $\mathrm{F}_{2} \alpha$ com o intuito de promover a luteólise de um eventual corpo lúteo persistente, não detectável à palpação trans-retal. Foi realizada a dosagem de progesterona plasmática pela técnica modificada de radioimunoensaio (ABRAHAM et al., 1971) das éguas que permaneceram acíclicas durante toda a EM, para confirmação laboratorial da condição de anestro.

Para o estudo das características foliculares, usou-se o método dos quadrados mínimos, procedimento General Linear Models (GLM) do pacote estatístico SAS (SAS, 2001).

O estudo da dinâmica folicular, nos nove dias que precederam cada ovulação, foi feito considerando-se as seguintes características: NFPEQ, NFMED, NFGRA, NFOLS e MAIORF, utilizando-se do modelo a seguir: $\mathrm{Y}_{\mathrm{ijkl}}=\mu+\mathrm{M}_{\mathrm{i}}+$ $\mathrm{Ej}+\mathrm{L}_{\mathrm{k}}+\mathrm{b} 1(\mathrm{Xl}-\mathrm{X})+$ eijkl, em que $\mathrm{Y}_{\mathrm{ijk} l}=$ valor observado para as características em cada palpação; $\mu=$ média geral; $\mathrm{M}_{\mathrm{i}}=$ efeito do i-ésimo mês da palpação $(\mathrm{i}=11,12,1,2,3) ; \mathrm{Ej}=\mathrm{j}$-ésima classe de condição corporal $(j=1,2,3) ; \mathrm{L}_{\mathrm{k}}=$ efeito da $\mathrm{k}$-ésima categoria reprodutiva da égua $(\mathrm{k}=1,2$ onde $\mathrm{K}=1$ é égua com potro ao pé e $\mathrm{k}=2$, solteira); $\mathrm{b} 1$ = coeficiente de regressão da diferença, em dias, entre a data da ovulação e a data da palpação, sobre a característica estudada; $\mathrm{Xl}=$ diferença, em dias, entre a data da ovulação e a data da palpação, sobre a característica estudada; $\mathrm{X}=$ valor médio de $\mathrm{Xl} ;$ eijkl = erro aleatório associado a cada observação em particular, ou seja, l-ésima palpação.

As parcelas não significativas no modelo geral foram posteriormente omitidas do mesmo para simplificação, originando outros modelos reduzidos, a saber: 1) para o estudo da dinâmica folicular durante a estação de monta, foram consideradas as características ovarianas anteriormente citadas, utilizando-se o seguinte modelo: $Y_{i j k l}=\mu+M_{i}+E j+L_{k}+$ eijkl; 2) para o estudo da época da primeira ovulação na estação de monta, considerou-se a característica condição corporal de novembro, utilizando-se o seguinte modelo: $Y_{i j}=\mu+E_{i}+$ eij.

A significância dos fatores estudados (mês da palpação, condição corporal e categoria reprodutiva) foi avaliada pelo teste estatístico de Student-Newman-Keuls (SNK). As variáveis não paramétricas foram comparadas em tabelas de contingência por teste do qui-quadrado $\left(\lambda^{2}\right)$, segundo SAMPAIO (1998).

\section{RESULTADOS E DISCUSSÃO}

Das 134 reprodutoras avaliadas, 99 $(73,9 \%)$ apresentaram atividade ovariana cíclica durante a EM, enquanto $35(26,1 \%)$ permaneceram em anestro. Os resultados da dosagem de progesterona plasmática confirmaram a condição de anestro em razão das concentrações inferiores a $1 \mathrm{ng} / \mathrm{mL}$. A CC média ao início da EM das éguas acíclicas foi $3,9 \pm 1,1$ e das cíclicas, $4,3 \pm 1,0$ $(\mathrm{P}>0,05)$.

A taxa de gestação do plantel foi $56,7 \%$ (76/134); entretanto, computando-se apenas as éguas com atividade ovariana cíclica, a mesma foi 77\% (76/99). A taxa de gestação foi afetada pelo mês de ocorrência da ovulação $(\mathrm{P}<0,05)$, sendo que o maior índice foi observado no mês de fevereiro $(71,1 \%)$, não diferindo nos meses de janeiro $(50,0 \%)$ e março $(59,1 \%)$. As menores taxas de gestação foram obtidas nos meses de novembro $(12,5 \%)$ e dezembro $(29,6 \%)$, com a ressalva da EM ter se iniciado em 15 de novembro.

As taxas de gestação/ciclo foram $75,5 \%$ (37/49), 88,9\% (24/27), 76,5\% (13/17) e 40,0\% (2/5) para o primeiro, segundo, terceiro e quarto ciclos, respectivamente. $O$ número de ciclos/concepção foi $2,3(174 / 76)$ e o número de saltos/ciclo foi 2,1 (361/174). Após serem testadas as taxas de gestação à monta natural $(\mathrm{MN})$, não se constatou diferença significativa $(\mathrm{P}>0,05)$ entre os reprodutores utilizados.

Tanto a CC obtida ao início do experimento (agosto) como ao início da EM (novembro), não foram boas indicadoras de quais éguas apresentariam atividade cíclica ou não no seu decorrer, visto que a distribuição das éguas cíclicas não diferiu $(\mathrm{P}>0,05)$ entre as classes de 
Eficiência reprodutiva e dinâmica folicular de éguas Campolina...

condição corporal. No entanto, ao se considerar a evolução da CC, pôde-se observar seu efeito

$(\mathrm{P}<0,05)$ sobre o número de éguas que ciclaram na EM (Tabela 1).

Tabela 1 - Frequência de éguas cíclicas durante a estação de monta, segundo a evolução da condição corporal desde o início da estação de monta até a última ovulação

\begin{tabular}{lcc}
\hline Evolução da condição & \multicolumn{2}{c}{ Éguas cíclicas } \\
\cline { 2 - 3 } corporal & $\mathrm{n}$ & $\%^{*}$ \\
\hline Ganhou & $58 / 69$ & $84,1^{\mathrm{a}}$ \\
Manteve & $9 / 17$ & $47,1^{\mathrm{b}}$ \\
Perdeu & $6 / 11$ & $54,6^{\mathrm{ab}}$ \\
\hline
\end{tabular}

*a,bvalores dentro da coluna seguidos de letras diferentes diferem entre si $(\mathrm{P}<0,05)$.

Não foi constatado efeito significativo da CC de novembro sobre a época de ocorrência da primeira ovulação $(\mathrm{P}>0,05)$; no entanto, observou-se que as éguas da classe 3 (escore $\geq 5$ ) ovularam, em média, 12,1 e 9,8 dias antes das éguas das classes 1 (escore $\leq 3,5$ ) e 2 (escore $4-4,5$ ), respectivamente.

A CC mais próxima da última palpação $(\mathrm{CC}$ obtida no intervalo de \pm 30 dias da última ovulação na EM) e a evolução da CC tiveram efeito sobre a taxa de gestação, pois as éguas da classe 1 obtiveram menores taxas de prenhez quando comparadas àquelas das classes 2 e $3(\mathrm{P}<0,05)$. Da mesma forma, os animais que perderam $\mathrm{CC}$ apresentaram menor taxa de gestação, a qual diferiu $(\mathrm{P}<0,05)$ daquelas obtidas em éguas que mantiveram ou ganharam CC (Tabela 2).

$\mathrm{O}$ mês teve efeito $(\mathrm{P}<0,05)$ sobre todas as características foliculares estudadas, sendo que os valores médios do NFPEQ, NFMED e NFOLS foram maiores no mês de março e a média do NFGRA foi superior no mês de dezembro, considerando-se apenas os meses da EM. O diâmetro médio do maior folículo foi superior em dezembro $(54,0 \mathrm{~mm})$, em contraste com março $(45,4 \mathrm{~mm})$, mês em que foram encontrados os menores valores.

Com exceção da característica folicular NFGRA, as demais foram afetadas pela condição corporal (Tabela 3).

Tabela 2 - Taxa de gestação de éguas mantidas sob manejo extensivo de criação, de acordo com a condição corporal mais próxima da última ovulação e a evolução da condição corporal

\begin{tabular}{|c|c|c|c|c|c|c|}
\hline \multirow[b]{2}{*}{ Variável } & \multicolumn{3}{|c|}{ 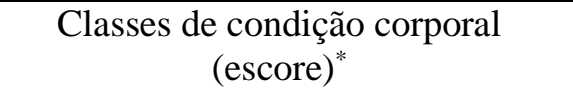 } & \multicolumn{3}{|c|}{$\begin{array}{c}\text { Evolução da } \\
\text { condição corporal }\end{array}$} \\
\hline & $1(\leq 3,5)$ & $2(4-4,5)$ & $3(\geq 5)$ & Perdeu & Manteve & Ganhou \\
\hline Taxa de gestação (\%) & $7,1^{\mathrm{a}}$ & $51,2^{\mathrm{b}}$ & $63,6^{\mathrm{b}}$ & $11,1^{\mathrm{a}}$ & $43,8^{b}$ & $63,9^{\mathrm{b}}$ \\
\hline
\end{tabular}

Tabela 3 - Número médio ( \pm desvio padrão) de folículos pequenos, médios, grandes, total e diâmetro do maior folículo, de acordo com a classe de condição corporal, durante a estação de monta de éguas

\begin{tabular}{lccccc}
\hline Classes de & \multicolumn{5}{c}{ Média \pm desvio padrão } \\
\cline { 2 - 6 } $\begin{array}{l}\text { Condição } \\
\text { Corporal }\end{array}$ & NFPEQ $^{1}$ & NFMED $^{2}$ & NFGRA $^{3}$ & NFOLS $^{4}$ & MAIORF $^{5}$ \\
\cline { 2 - 5 } & $\leq 15 \mathrm{~mm}$ & $>15<35 \mathrm{~mm}$ & $\geq 35 \mathrm{~mm}$ & $\mathrm{n}$ & $\mathrm{mm}$ \\
\hline $1(\leq 3,5)$ & $2,6 \pm 0,6^{\mathrm{b}^{*}}$ & $0,9 \pm 0,2^{\mathrm{b}}$ & $0,5 \pm 0,1^{\mathrm{a}}$ & $4,0 \pm 0,9^{\mathrm{b}}$ & $5,3 \pm 1,2^{\mathrm{a}}$ \\
$2(4-4,5)$ & $3,0 \pm 0,8^{\mathrm{a}}$ & $1,2 \pm 0,3^{\mathrm{a}}$ & $0,5 \pm 0,1^{\mathrm{a}}$ & $4,7 \pm 1,1^{\mathrm{a}}$ & $4,9 \pm 1,1^{\mathrm{b}}$ \\
$3(\geq 5)$ & $2,9 \pm 0,7^{\mathrm{a}}$ & $1,3 \pm 0,3^{\mathrm{a}}$ & $0,6 \pm 0,1^{\mathrm{a}}$ & $4,8 \pm 1,0^{\mathrm{a}}$ & $5,0 \pm 1,0^{\mathrm{b}}$ \\
\hline
\end{tabular}

$* \mathrm{a}, \mathrm{b}$ valores dentro da linha seguidos de letras diferentes diferem entre si $(\mathrm{P}<0,05)$.

${ }^{1} \mathrm{NFPEQ}=$ número de folículos pequenos; ${ }^{2} \mathrm{NFMED}=$ número de folículos médios; ${ }^{3} \mathrm{NFGRA}=$ número de folículos grandes;

${ }^{4} \mathrm{NFOLS}=$ número total de folículos $(\mathrm{NFOLS}=\mathrm{NFPEQ+NFMED+NFGRA}) ;{ }^{5} \mathrm{MAIORF}=$ diâmetro do maior folículo. 
De acordo com o efeito do dia da palpação sobre as características foliculares observou-se que o NFGRA não variou nos nove dias precedentes à ovulação, já o NFPEQ, NFMED e NFOLS diminuíram $(\mathrm{P}<0,05)$ à medida que a ovulação se aproximava, enquanto o MAIORF aumentou $(\mathrm{P}<0,05)$ do dia -9 ao dia 0 (dia da ovulação).

A CC teve efeito sobre o NFMED e NFOLS $(\mathrm{P}<0,05)$ no período pré-ovulatório, pois as éguas da classe 1 apresentaram valores superiores $(0,9$ e 4,1, respectivamente) em relação às éguas das classes 2 $(0,7$ e 3,8$)$ e $3(0,8$ e 3,8$)$. Não foi observado efeito significativo da CC sobre o NFPEQ, NFGRA e MAIORF.

Dentre as éguas acíclicas, $71,4 \%$ tinham potro ao pé e $28,6 \%$ eram não lactantes. A má $\mathrm{CC}$ ao início (escore $=3,9 \pm 1,1$ ) e ao final (escore $=4,1 \pm$ $0,8)$ da EM parece ter sido fator determinante da alta percentagem de animais em anestro, pois a lactação e a presença do potro, quando as fêmeas possuem uma boa CC, como relatado por NAGY et al. (1998), não interferem na atividade ovariana cíclica de éguas. $\mathrm{O}$ anestro lactacional, segundo a literatura, não ocorre na espécie equina (NAGY et al., 1998; DEICHSEL \& AURICH, 2005), porém algumas éguas podem apresentar apenas o cio do potro e a seguir entrarem em anestro (PALMER \& DRIANCOURT, 1983). GODOI et al. (2002) relatam que o parto teve efeito positivo sobre a foliculogênese de éguas lactantes, pois as mesmas apresentaram maior diâmetro do folículo pré-ovulatório e maior número de ondas foliculares que éguas não lactantes; da mesma forma, os resultados demonstraram o importante papel que a $\mathrm{CC}$ exerce sobre a atividade ovariana, pois éguas que ganharam peso apresentaram maior diâmetro do folículo pré-ovulatório e um menor intervalo entre o parto e a primeira ovulação.

A elevada taxa de anestro afetou negativamente a taxa de gestação do rebanho, que foi $56,7 \%$. Ao se considerar somente as éguas cíclicas, tem-se uma taxa de prenhez de $77 \%$, índice inferior aos obtidos por SERENO et al. (1997) e ZÚCCARI et al. (2002), porém superior aos relatados por LIMA et al. (2000) e MOREL \& GUNNARSSON (2000).

Os baixos valores encontrados para a taxa de gestação nos dois primeiros meses da EM podem ser explicados pelos baixos índices pluviométricos nos meses de setembro e outubro, que somaram apenas 4,4 $\mathrm{mm}$, valor muito abaixo do esperado para a época das águas, fato que fez com que as éguas perdessem e/ou permanecessem em má $\mathrm{CC}$, em razão da baixa disponibilidade e má qualidade das pastagens. Já as maiores taxas de gestação, nos últimos meses da EM, foram reflexo da maior precipitação pluviométrica nos meses de novembro e dezembro (410,7 mm), o que resultou em melhora substancial das pastagens e da CC dos animais. De fato, HENNEKE et al. (1984) relataram que éguas com escore de $\mathrm{CC}<5$ apresentaram maiores intervalos entre o início da EM e a ocorrência do primeiro cio e da ovulação, quando comparadas àquelas com $\mathrm{CC}>5$. Nesse sentido, GASTAL et al. (2004) observaram maior incidência de várias ondas anovulatórias nas éguas com má condição corporal que naquelas com boa condição corporal; assim, o período de transição que antecede a primeira ovulação da estação de monta foi maior para éguas em má condição corporal.

Embora os resultados de taxa de gestação/ciclo sejam similares aos da literatura (VIVO et al., 1985; SATURNINO et al., 2002; ZÚCCARI et al., 2002), acredita-se que o percentual para o primeiro ciclo, provavelmente, poderia ter sido maior, caso não tivesse ocorrido uma precipitação pluviométrica tão baixa, pois foi a época em que ocorreram $41,4 \%$ das ovulações (novembro e dezembro).

O número de ciclos/concepção $(2,3)$ encontra-se acima dos valores obtidos em outros trabalhos (HENNEKE et al., 1984; CAMILLO et al., 1997; SATURNINO et al., 2002; ZÚCCARI et al., 2002), em decorrência dos baixos índices de gestação apresentados nos primeiros 45 dias da EM.

A evolução da CC das éguas no transcorrer da EM mostrou-se efetiva, pois esse ganho teve reflexo positivo sobre o percentual de animais em atividade cíclica, confirmando dados da literatura (HENNEKE et al., 1984; NAGY et al., 1998; MCMANUS \& FITZGERALD, 2000; GODOI et al., 2002).

Parece provável que durante as avaliações da CC, ao início da EM, o escore possa ter sido superestimado. Tal fato talvez seja a causa de não se ter observado efeito da CC sobre a época de ocorrência da primeira ovulação, o que era esperado, a partir dos resultados de NAGY et al. (1998), GASTAL et al. (2004), FERREIRA-DIAS et al. (2005) e VECCHI et al. (2010).

Durante a EM, a dinâmica folicular caracterizou-se por uma frequência aproximada de $60 \%$ de folículos pequenos, $20 \%$ de folículos médios e $15 \%$ de folículos grandes, exceto nos meses de novembro e dezembro, quando a frequência de folículos grandes aumentou para $25 \%$ em detrimento dos médios. Isso caracterizou uma dinâmica maior nos primeiros meses da EM, de forma semelhante ao obtido por PIERSON \& GINTHER (1987).

No estudo da dinâmica folicular, nos nove dias precedentes à ovulação, observou-se efeito de dia $(\mathrm{P}<0,05)$ sobre as características foliculares 
Eficiência reprodutiva e dinâmica folicular de éguas Campolina...

estudadas, exceto para o número de folículos grandes, o que era esperado, uma vez que essa categoria compreendia folículos com diâmetro maior que $35 \mathrm{~mm}$, correspondendo, portanto, ao folículo pré-ovulatório. Os dados observados são similares àqueles de GINTHER (2000), GINTHER et al. (2001), DRIANCOURT (2001), BEG \& GINTHER (2006) e GURGEL et al. (2008).

O diâmetro médio do maior folículo préovulatório não diferiu significativamente entre as classes de condição corporal, em contraste com os resultados encontrados por GODOI et al. (2002), GASTAL et al. (2004) e RODRIGUES et al. (2011), que demonstraram que o diâmetro do maior folículo pré-ovulatório diferiu significativamente entre éguas com boa $\mathrm{CC}$ em detrimento dos animais com má condição corporal.

À semelhança dos resultados de GENTRY et al. (2002ab) e GASTAL et al. (2004), a CC teve efeito sobre as características foliculares estudadas, sendo que as éguas com melhor CC apresentaram uma população de folículos superior àquelas em baixa CC. Possivelmente, essa maior atividade ovariana seja um dos fatores que colaboraram para a obtenção de maior percentual de éguas cíclicas e gestantes entre os animais com boa CC. Sob o aspecto endocrinológico, os achados de HOUSEKNECHT et al. (1998), BUFF et al. (2002) e FERREIRA-DIAS et al. (2005) indicaram que uma baixa CC pode comprometer a função do eixo hipotálamo-hipófise-gônadas e o controle do mecanismo hormonal que desencadeia a atividade folicular estaria comprometido nesses animais.

\section{CONCLUSÃO}

Nas condições de campo em que o estudo foi realizado, constatou-se que, no decorrer da estação de monta, a população de folículos variou nos nove dias precedentes à ovulação, havendo, da mesma forma, efeito do mês da estação de monta sobre as características foliculares. Para as éguas mantidas sob o manejo extensivo de criação, a precipitação pluviométrica foi o fator ambiental com papel preponderante sobre o escore de condição corporal que, por sua vez, teve efeito sobre a ocorrência de atividade cíclica, a dinâmica folicular e a taxa de gestação.

\section{REFERÊNCIAS}

ABRAHAM, G.E.; SWERDLOFF, R.; TULCHINSKY, D.; ODELL, W.D. Radioimunoassay of plasma progesterone. The Journal of Clinical Endocrinology \& Metabolism, v.32, p.619, 1971.
BEG, M.A.; GINTHER, O.J. Follicle selection in cattle and horse: role of intrafollicular factors. Society of Reproduction and Fertility, v.132, p.365-377, 2006.

BUFF, P.R.; DODDS, A.C.; MORRISON, C.D.; WHITLEY, N.C.; MCFADIN, E.L.; DANIEL, J.A.; DJIANE, J.; KEISLER, D.H. Leptin in horses: tissue localization and relationship between peripheral concentrations of leptin and body condition. Journal of Animal Science, v.80, p.2942-2948, 2002.

CAMILLO F.; MARMORINI, P.; ROMAGNOLI, S.; VANNOZZI, I.; BAGLIACCA, M. Fertility of the first post partum estrous compared with fertility at the following estrous cycles in foaling mares and with fertility in nonfoaling mares. Journal of Equine Veterinary Science, v.17, p.612-616, 1997.

CARTER, R.A.; GEOR, R.J.; STANIAR, W.B.; CUBITT, T.A.; HARRIS, P.A. Apparent adiposity assessed by standardized scoring systems and morphometric measurement in horses and ponies. Veterinary Journal, v.179, p.204-210, 2009.

CAVINDER, C.A.; VOGELSANG, M.M.; GIBBS, P.G.; FORREST, D.W.; SCHMITZ, D.G. Endocrine profile comparisons of fat versus moderately conditioned mares following parturition. Journal of Equine Veterinary Science, v.7, n.2, p.72-79, 2007.

DEICHSEL, K.; AURICH, J. Lactation and lactational effects on metabolism and reproduction in the horse mare. Livestock Production Science, v.98, p.25-30, 2005.

DRIANCOURT, M.A. Regulation of ovarian follicular dynamics in farm animals: implications for manipulation of reproduction. Theriogenology, v.55, p.1211-1239, 2001.

FERREIRA-DIAS, G.; CLAUDINO, F.; CARVALLHO, H.; AGRÍCOLA, R.; ALPOIM-MOREIRA, J.;SILVA, J.R. Seasonal reproduction in the mare: possible role of plasma leptin, body weight and immune status. Domestic Animal Endocrinology, v.29, p.203-213, 2005.

GASTAL, M.O.; GASTAL, E.L.; SPINELLI, V.; GINTHER, O.J. Relationship between body condition and follicle development in mares. Animal Reproduction, v.1, n.1, p.115-121, 2004.

GENTRY, L.R.; THOMPSON, Jr., D.L.; GENTRY, Jr., G.T., DAVIS, K.A., GODKE, R.A.; CARTMILL, J.A. The relationship between body condition, leptin and reproductive and hormonal characteristics of mares during the seasonal anovulatory period. Journal of Animal Science, v.80, p.2695-2703, 2002a.

GENTRY, L.R.; THOMPSON, Jr., D.L.; GENTRY, Jr., G.T., DAVIS, K.A., GODKE, R.A. High versus low body condition in mares: Interactions with responses to somatotropin, GnRH analog and dexamethasone. Journal of Animal Science, v.80, p.3277-3285, 2002 b.

GENTRY, L.R.; THOMPSON, Jr., D.L.; GENTRY, Jr., G.T.; DEL-VECCHIO, R.P.; DAVIS, K.A.; DELVECCHIO, P.M. The relationship between body condition 
score and ultrasonic fat measurements in mares of high versus low body condition. Journal of Equine Veterinary Science, v.24, p.198-203, 2004.

GINTHER, O.J. Selection of the dominant follicle in cattle and horses. Animal Reproduction Science, v.60, p.61-79, 2000 .

GINTHER, O.J.; BEG, M.A.; BERGFELT, D.R.; DONADEU, F.X.; KOT, K.. Follicle selection in monovular species. Biology of Reproduction, v.65, p.638-647, 2001.

GODOI, D.B.; GASTAL, E.L.; GASTAL, M.O. A comparative study of follicular dynamics between lactating and non-lactating mares: effect of the body condition. Theriogenology, v.58, p.553-556, 2002.

GURGERL, J.R.C.; VIANA, C.H.C.; PEREZ, E.G.A.; NICHI, M. Dinâmica follicular em éguas: aspectos intrafoliculares. Revista Brasileira de Reprodução Animal, v.32, n.2, p.122-132, 2008.

HENNEKE, D.R.; POTTER, G.D.; KREIDER, J.L. Body condition during pregnancy and lactation and reproductive efficiency of mares. Theriogenology, v.21, n.6, p. $897-$ 909, 1984.

HENNEKE, D.R.; POTTER, G.D.; KREIDER, J.L.; YEATES, B.F. Relationship between condition score, physical measurements and body fat percentage in mares. Equine Veterinary Journal, v.15, n.4, p.371-372, 1983.

HOUSEKNECHT, K.L.; BAILE, C.A.; MATTERI, R.L.; SPURLOCK, M.E. The biology of leptin: a review. Journal of Animal Science, v.76, p.1505-1420, 1998.

KENNEY, R.M.; BERGMAN, R.V.; COOPER, W.L.; MORSE, G.W. Minimal contamination on techniques of breeding mares: Technique and preliminary findings. Proceedings of American Association of Equine Practitioners, v.21, p.327-336, 1975.

KÖPPEN, W. Climatologia. México, Fondo de Cultura Economica, 1931. 2v.

LIMA, M.C.C.; SILVA FILHO, J.M.; CARVALHO, G.R.; PALHARES, M.S.; VALLE, G.R.; OLIVEIRA H.N. Efeito do número de inseminações artificiais por ciclo sobre a fertilidade de éguas inseminadas com sêmen equino diluído, resfriado a $20^{\circ} \mathrm{C}$ e transportado. Revista Brasileira de Zootecnia, v.29, n.6, p.1649-1653, 2000.

McMANUS, C.J.; FITZGERALD, B.P. Effects of a single day of feed restriction on changes in serum leptin, gonadotropins, prolactin, and metabolites in aged and young mares. Domestic Animal Endocrinology, v.19, p.1-13, 2000.

MOREL, M.C.G.D.; GUNNARSSON, V. A survey of the fertility of Icelandic stallions. Animal Reproduction Science, v.64, p.49-64, 2000.

NAGY, P.; HUSZENICZA, G.; JUHÁSZ, J.; KULCSÁR, M.; SOLTI, L.; REICZIGEL, J.; ABAVÁRY, K. Factors influencing ovarian activity and sexual behavior of postpartum mares under farm conditions. Theriogenology, v.50, p.1109-1019, 1998.

PALMER, E.; DRIANCOURT, M.A. Some interactions of season of foaling, photoperiod and ovarian activity in the equine. Livestock Production Science, v.10, p.197$210,1983$.

PEUGNET, P.; DUCHAMP, G.; REIGNER, F.; DUPONT, J.; BARREY, E.; GUILLAUME, D. Effect of growth hormone treatment on follicular growth in well-fed or feed-restricted mares. Animal Reproduction Science, v.1215, p.42-44, 2010.

PIERSON, R.A.; GINTHER, O.J. Follicular population dynamic during the estrous cycle of the mare. Animal Reproduction Science, v.14, n.3, p.219-231, 1987.

RODRIGUES, P.G.; RAYMUNDO, C.M.; SOUZA, J.C.; MIRANDA, M.C.M.G.; REZENDE, A.S.C. Gordura corporal e eficiência reprodutiva em éguas doadoras de embrião Mangalarga Marchador. Ciência e Agrotecnologia, v.5, n.5, p.1002-1008, 20111.

SAMPAIO, I.B.M. Estatística aplicada à experimentação animal. Fundação de Ensino e Pesquisa em Medicina Veterinária, Belo Horizonte, 1998, 221 p.

Statistical Analysis System - SAS. SAS/STAT software, Version 8.02, Cary: 2001.

SATURNINO, H.M.; SILVA FILHO J.M.; SILVA M.D.; PALHARES, M.S.; BRANDÃO, F.Z.; OLIVEIRA, H.N. Efeito do intervalo das duas últimas inseminações sobre a fertilidade de éguas inseminadas com sêmen fresco diluído. Revista Brasileira de Zootecnia, v.31, n.3, p.1143-1149, 2002.

SERENO, J.R.B.; SANTOS, S.A.; ZÚCCARI, C.E.S.N.; MAZZA, M.C.M. Establishment of a breed season and evaluation of the reproductive performance of the Pantaneira horse in Mato Grosso do Sul, Brasil. Animal Genetic Resource Information, v.21, p.43-48, 1997.

VECCHI, I.; SABBIONI, A.; BIGLIARDI, E.; MORINI, G.; FERRARI, L.; DI CIOMMO, F.; SUPERCHI P.; PARMIGIANI, E.. Relationship between body fat and body condition score and their effects on estrous cycles of Standarbreed maiden mare. Veterinary Research Communication, v.34 (suppl. 1), p.41-45, 2010. Disponível

em

http://link.springer.com/article/10.1007/s11259-010-9407$\underline{0}$ acesso em out. 2013.

VIVO, R.; SANTISTEBAN, R.; TOVAR, P.B.; CASTEJÓN, M.F. Valores de fertilidad em yeguas espanolas y árabes. Archivos de Zootecnia, v.34, n.129, p.159-167, 1985.

ZÚCCARI, C.E.S.N.; NUNES, D.B.; CORRÊA FILHO, R.A.C. Eficiência reprodutiva de éguas da raça Pantaneira durante as estações de monta 1995/2000. Archivos de Zootecnia, v.51, p.139-148, 2002. 